\title{
Potential energy curves of molecular nitrogen for singly and doubly ionized states with core and valence holes
}

\author{
Debarati Bhattacharya, ${ }^{*} \dagger$ K. R. Shamasundar, ${ }^{\ddagger}$ and Agapi Emmanouilidou ${ }^{\dagger}$ \\ $\dagger$ Department of Physics and Astrophysics, University College London, Gower Street, London \\ WC1E $6 B T, U K$ \\ $\ddagger$ Indian Institute of Science Education and Research, Mohali, Sector 81, SAS Nagar 140306, \\ India \\ E-mail: d.bhattacharya@ucl.ac.uk
}

\begin{abstract}
Theoretical description of potential energy curves (PECs) of molecular ions is essential for interpretation and prediction of coupled electron-nuclear dynamics following ionization of parent molecule. However, an accurate representation of these PECs for core or inner valence ionized state is non-trivial, especially at stretched geometries for double or triple bonded systems. In this work, we report PECs of singly and doubly ionized states of molecular nitrogen using state-of-the-art quantum chemical methods. The valence, inner valence and core ionized states have been computed. A double loop optimisation scheme that separates the treatment of the core and the valence orbitals during the orbital optimization step of the multi-configuration self-consistent field method has been implemented. This technique allows the energy to be converged to any desired ionized state with any number of core or inner shell holes. The present work also compares the PECs obtained using both delocalized and localized set of orbitals for
\end{abstract}


the core-hole states. The PECs of a number of singly and doubly ionized valence states have also been computed and compared with previous studies. The computed PECs reported here are expected to be of importance for future studies to understand the interplay between photoionization and Auger spectra during the break-up of molecular nitrogen when interacting with intense free electron lasers.

\section{Introduction}

The advancement of experimental techniques in $\mathrm{X}_{\text {-ray }}{ }^{1}$ and extreme ultraviolet spectroscopy ${ }^{2,3}$ has facilitated an in depth study of electron dynamics in atomic ${ }^{4,5}$ and molecular systems ${ }^{6,7}$ as well as coupled electron-nuclear dynamics in molecules. ${ }^{8,9}$ Accurate theoretical methods are needed to interpret and utilise the results obtained from high precision XUV measurements. Intense X-ray sources, e.g., synchrotrons or X-ray free electron lasers (FEL) are capable of inducing ionization from inner-shell electrons of atoms and molecules. The ionization of inner shell electrons gives rise to an interplay between ionization by single photon absorption and Auger processes. Most studies addressing the interaction of molecules with FEL pulses do not account for the nuclear motion. This is due to the difficulty involved in computing the potential energy curves (PEC) for molecules with core hole states. The dissociation of the molecule is taken into account mostly in a phenomenological way through additional terms

in the rate equations employed to compute the resulting atomic ion yields. ${ }^{10}$ In this work, we compute PECs of singly and doubly ionized states of molecular nitrogen. This will allow future studies to explicitly account for the nuclear motion when a molecule interacts with a FEL-pulse. ${ }^{11,12}$

Several theoretical studies have computed PECs of singly and doubly ionized states of $\mathrm{N}_{2}$ employing quantum chemistry packages. ${ }^{13-17}$ These studies compute the PECs for $\mathrm{N}_{2}$ states with one or two outer valence electrons missing or with one core hole. These computations have been carried out using a multi-configurational self consistent field (MCSCF) method. ${ }^{18-20}$ To achieve better accuracy for the PECs of states with outer valence holes, in addition to 
MCSCF, some theoretical studies employ the multi-reference configuration interaction (MRCI) method. ${ }^{21-23}$ In this work, for completeness, we also compute the PECs of $\mathrm{N}_{2}$ states with one or two outer valence electrons missing. In contrast, there are a few studies computing the PECs for $\mathrm{N}_{2}$ states with an inner valence ${ }^{24}$ or core electron missing. ${ }^{25-27}$ The computation of the PECs of these latter states is not trivial. The reason is that there are several states with lower energy that have the same symmetry as the state of interest that has an inner valence or core hole. This results in variational collapse ${ }^{28}$ to the lowest energy state during optimization of the orbitals and the coefficients in methods like MCSCF. ${ }^{28,29}$ In previous studies, the computation of the PECs of $\mathrm{N}_{2}$ with a single core hole ${ }^{25-27}$ has been carried out in a two-step optimization process. In the first step, the valence orbitals are optimized with MCSCF, while the core orbitals are kept frozen. In the second step, it is the valence orbitals that are kept frozen, while we optimize with MCSCF the core ones. Such a two-step process was implemented by Rocha ${ }^{30}$ to obtain the PECs for the ionized states of the CO molecule. A similar two-step optimisation process has also been employed by Carravetta et $a l^{31}$ to compute the energy at the equilibrium internuclear distance, rather than PECs, of $\mathrm{N}_{2}$ states with multiple core holes. Herein, first, we compute the PECs of $\mathrm{N}_{2}$ states with one or two outer valence electrons missing. We do so for completeness and to compare our results with existing ones. Most importantly, employing this two-step optimization process, we also compute the PECs of $\mathrm{N}_{2}$ states with one inner valence hole or two core hole states. To our knowledge, currently, there are no studies of the PECs for these latter singly and doubly ionized states of $\mathrm{N}_{2}$.

\section{Methods}

In what follows, we describe the computation of the PECs of singly and doubly ionized states of $\mathrm{N}_{2}$ that involve all possible combinations of electrons missing either from valence or

core orbitals. The ground state electronic configuration of $\mathrm{N}_{2}$ is $\left(1 \sigma_{g}^{2} 1 \sigma_{u}^{2} 2 \sigma_{g}^{2} 2 \sigma_{u}^{2} 1 \pi_{u x}^{2} 1 \pi_{u y}^{2}\right.$ 
$\left.3 \sigma_{g}^{2}\right)$. The $1 \sigma_{g}$ and $1 \sigma_{u}$ are core orbitals, $2 \sigma_{g}$ is an inner valence orbital, ${ }^{32}$ while the rest are referred to as valence ones. The $\mathrm{MCSCF}^{33}$ method combines the configuration interaction (CI) with the self-consistent field (SCF) method. The former optimizes the coefficients of the Slater determinants, while SCF optimizes the orbitals involved in the Slater determinants. Moreover, $\mathrm{MCSCF}^{33-36}$ involves a combination of Slater determinants that account for all possible electronic excitation of the molecule under consideration.

In the current work, we employ the complete active space (CAS) variant of the MCSCF method, which we refer to as CASSCF. ${ }^{37,38}$ The CASSCF method involves active orbitals with an occupancy, $n$, that ranges from $0<n<2$, fully occupied closed-shell inactive orbitals, and fully unoccupied inactive virtual orbitals. In the current work, we consider 10 active orbitals $1 \sigma_{g}, 1 \sigma_{u}, 2 \sigma_{g}, 2 \sigma_{u}, 1 \pi_{u x}, 1 \pi_{u y}, 3 \sigma_{g}, 1 \pi_{g x}, 1 \pi_{g y}, 3 \sigma_{u}$, where the first seven correspond to the nearly doubly occupied orbitals in the ground state of $\mathrm{N}_{2}$ around equilibrium geometry and the last three have a weaker occupancy. The three virtual orbitals that we select as active orbitals are the lowest in energy and have similar energies, all other virtual orbitals have much higher energies. Hence, the selection is all electron core and full valence active space. This CASSCF method allows for an accurate computation of the energy of an ionized state of $\mathrm{N}_{2}$ as a function of the internuclear distance, since it accounts for all possible electronic excitations to three virtual orbitals. Here, we perform the $\mathrm{CASSCF}^{39,40}$ calculations using Molpro, ${ }^{41,42}$ which is a quantum chemistry package. In particular, we employ the standard release version MOLPRO2020.1. To improve the initial description of the orbitals of ionized states of $\mathrm{N}_{2}$, we run a CASSCF with ten active orbitals for the ground state of $\mathrm{N}_{2}$. In addition, for the computation of $\mathrm{N}_{2}$ states with one valence hole, we employ the augmented Dunning correlation consistent quadruple valence basis set (aug-cc-pVQZ), ${ }^{43}$ while we use the quintuple zeta basis set (cc-pV5Z) ${ }^{43}$ for states with two valence holes. Such basis sets have been previously employed to accurately account for electronic correlation in singly and doubly ionized states. ${ }^{23,24}$

Finally, to obtain the PECs of $\mathrm{N}_{2}$ states with one or two valence holes we optimize all 
ten orbitals with the CASSCF method. In contrast, we compute the PECs of $\mathrm{N}_{2}$ states with one or two core holes by employing a two-step optimization process, similar to the two-step process in Ref. 30. Specifically, first, we freeze the two core orbitals $1 \sigma_{g}$ and $1 \sigma_{u}$ and optimize with CASSCF the remaining eight active ones. Then, we freeze the five occupied orbitals optimized in the first step and optimize with CASSCF the remaining two core and three virtual orbitals $1 \pi_{g x}, 1 \pi_{g y}$ and $3 \sigma_{u}$. In our calculations, we have checked that it suffices to perform this two-step optimization process once or twice in order for the energy of the $\mathrm{N}_{2}$ ionized states under consideration to converge.

\section{Results and discussion}

In what follows, we discuss our results for the PECs, first, for singly ionized states and then for doubly ionized states of $\mathrm{N}_{2}$, with valence and/or core electrons missing.

\section{Singly ionized states}

\section{Valence states}

In Figure 1(a), we present the PECs that we obtain using the CASSCF method for the ground state (black-dashed) and the three lowest energy singly ionized states of $\mathrm{N}_{2}$. We obtain the PECs up to the distance corresponding to the dissociation limit of $4 \AA$ of $\mathrm{N}_{2}$. The latter states are obtained when removing an electron from the $3 \sigma_{g}$ (blue-dashed), $1 \pi_{u}$ (grey-dashed) or $2 \sigma_{u}$ (brown-dashed) molecular orbital. In what follows, we refer to these singly ionized states either as $3 \sigma_{g}^{-1}, 1 \pi_{u}^{-1}$ (x or y) and $2 \sigma_{u}^{-1}$ or by the symmetry of the respective state $\left(1^{2} \Sigma_{g}^{+}, 1^{2} \Pi_{u}, 1^{2} \Sigma_{u}^{+}\right)$according to the $D_{\infty h}$ symmetry point group. As expected, the PECs of the $3 \sigma_{g}^{-1}, 1 \pi_{u}^{-1}$ (x or y) and $2 \sigma_{u}^{-1}$ states lie above the PEC of the ground state of $\mathrm{N}_{2}$ and converge to the same dissociation limit. We find our results for these four states of $\mathrm{N}_{2}$ to be in good agreement with those reported by Nagy et $a l^{13}$ for internuclear distances in the

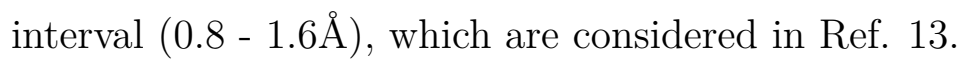




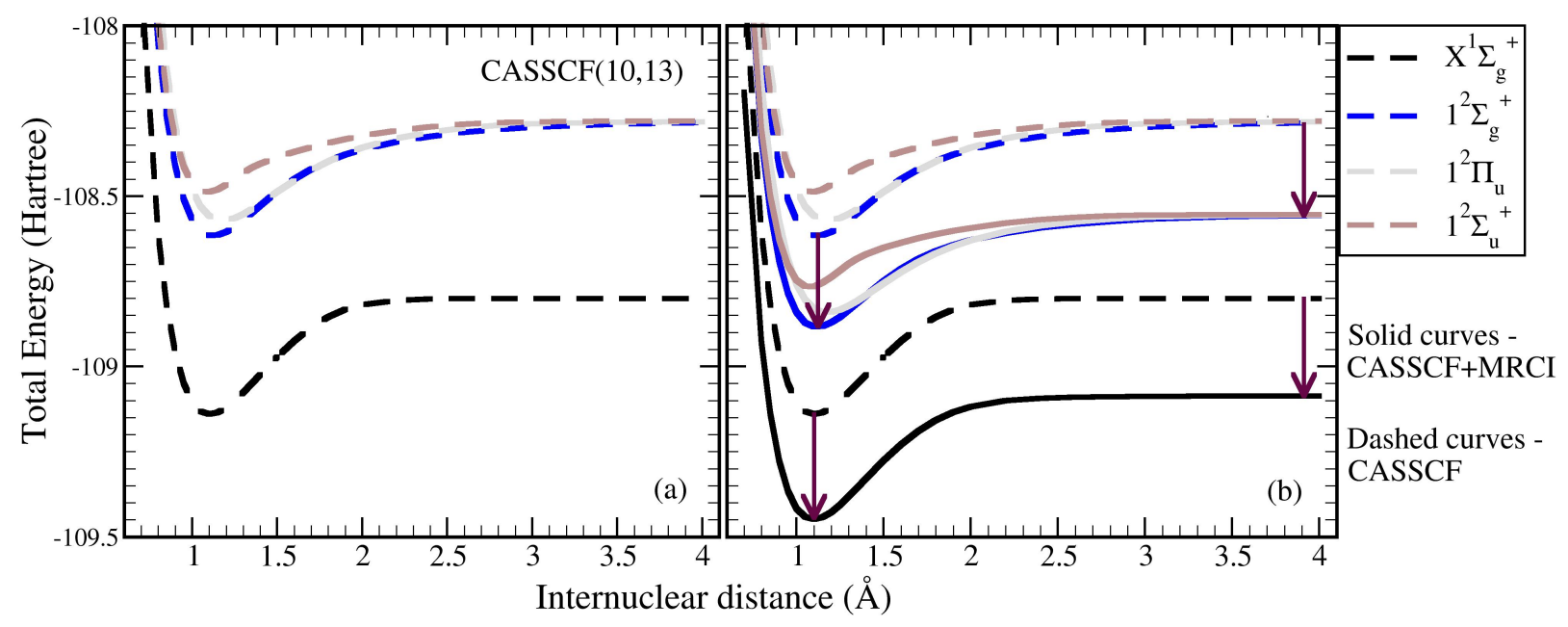

Figure 1: a) PECs for the ground state $\left(\mathrm{X}^{1} \Sigma_{g}^{+}\right)$and the three lowest energy singly ionized states of $\mathrm{N}_{2}$, i.e., $1^{2} \Sigma_{g}^{+}\left(3 \sigma_{g}^{-1}\right), 1^{2} \Pi_{u}\left(1 \pi_{u}^{-1}(\mathrm{x}\right.$ or y) $)$ and $1^{1} \Sigma_{u}^{+}\left(2 \sigma_{u}^{-1}\right)$. These curves are obtained with CASSCF. The total energy of each state is expressed in units of Hartree, while the internuclear distance is expressed in $\AA$. b) The PECs of the same four states of $\mathrm{N}_{2}$ as in a, obtained with CASSCF+MRCI (solid curves). For comparison, we also show the PECs obtained with CASSCF (dashed curves).

To obtain more accurate PECs for the ground and three lowest energy singly ionized states of $\mathrm{N}_{2}$, we employ the $\mathrm{MRCI}^{44-46}$ method using the optimized orbitals obtained from the CASSCF method and employing the same active space. The CASSCF includes all possible excitations only among active orbitals. The MRCI method allows single and double excitations from all closed-shell and active orbitals to all active and virtual orbitals. As a result, the MRCI method improves description of the electron-electron repulsion. Using Molpro, we have succeeded in applying MRCI after employing CASSCF for singly and doubly ionized states of $\mathrm{N}_{2}$ with holes in the outer valence orbitals. For singly and doubly ionized states with holes in core or inner valence orbitals we were not able to avoid variational collapse when applying MRCI following CASSCF. In Figure 1(b), we plot the thus obtained more accurate PECs of the four states of $\mathrm{N}_{2}$ with our results in Figure 1(a) obtained using only CASSCF. As expected, we find that the PECs obtained with the CASSCF+MRCI method have lower energies. However, we find that the equilibrium internuclear distance, the depth 
of the potential well and the dissociation limit of each PEC remains nearly the same when employing either the CASSCF or the CASSCF+MRCI methods. In addition, we find that our results for the PECs for the ground and three lower singly ionized states of $\mathrm{N}_{2}$ using the CASSCF+MRCI methods, are in excellent agreement with the results obtained in Ref. 22. We also find that the dissociation limit of the ground state of $\mathrm{N}_{2}$ is $9.82 \mathrm{eV}$ and of the three lowest singly ionized states is roughly equal to $24.3 \mathrm{eV}$, which are in very good agreement with the experimentally obtained ones of $9.78 \mathrm{eV}^{47}$ and $24.31 \mathrm{eV},{ }^{48}$ respectively.

Next, we compute the PEC of the $2 \sigma_{g}^{-1}$ singly ionized state of $\mathrm{N}_{2}$ resulting from the removal of an inner valence $\left(2 \sigma_{g}\right)$ electron. As already pointed out in the introduction, this is not a trivial task. The reason is that besides the desired state there are several other states with the same symmetry $\left(\Sigma_{g}^{+}\right)$and lower energy. To obtain the desired singly ionized state, we proceed as follows. During the CASSCF calculation, we ensure that Molpro ${ }^{41,42}$ computes a sufficient number of $\Sigma_{g}^{+}$symmetry states as a function of the internuclear distance. We identify the desired singly ionized state of $\mathrm{N}_{2}$ by selecting the $\Sigma_{g}^{+}$state that has an occupation number equal to 1 for the $2 \sigma_{g}$ state at the equilibrium geometry. We note that the symmetry of the states of $\mathrm{N}_{2}$ are described by the $D_{\infty h}$ symmetry point group. However, in Molpro, the states of linear homonuclear molecules are described by the reduced $D_{2 h}$ symmetry point group. As a result, for instance, an $A_{g}$ symmetry state in $D_{2 h}$ corresponds to more than one state, i.e., $\Sigma_{g}^{+}$or $\Delta_{g}$ in the $D_{\infty h}$ symmetry point group. In all our calculations in the current work, when the state considered in Molpro in the $D_{2 h}$ symmetry point group corresponds to more than one state in the $D_{\infty h}$ group, we also specify the $\Lambda$ quantum number in Molpro, referred to lquant. That is, for the $2 \sigma_{g}^{-1}$ state $\Lambda$ is set equal to zero in the input Molpro file employed in our calculations. We find that the $2 \sigma_{g}^{-1}$ state of $\mathrm{N}_{2}$ corresponds to the $6^{\text {th }}$ lowest energy state of $\Sigma_{g}^{+}$symmetry, in accord with Ref. 16. This state (red-dashed curve) is plotted in Figure. 2. For comparison, we also plot the ground and the three lowest energy singly excited states of $\mathrm{N}_{2}$. We find, that the $2 \sigma_{g}^{-1}$ state is repulsive in nature. This is in contrast to the other three PECs which have an energy minima as a function of the internuclear distance. 
In addition, we find that the vertical ionization energy from the ground state of $\mathrm{N}_{2}$ to the $2 \sigma_{g}^{-1}$ ionized state is $38.3 \mathrm{eV}$. This compares very well with the value of $40.5 \mathrm{eV}$ reported by Aoto et al. ${ }^{16}$ that employ a CASSCF method similar to the one employed in the current work. Our result for the vertical ionization energy also compares well with the value of 36.9 eV reported by Kornilov et al. ${ }^{24}$ that do not employ CASSCF method.

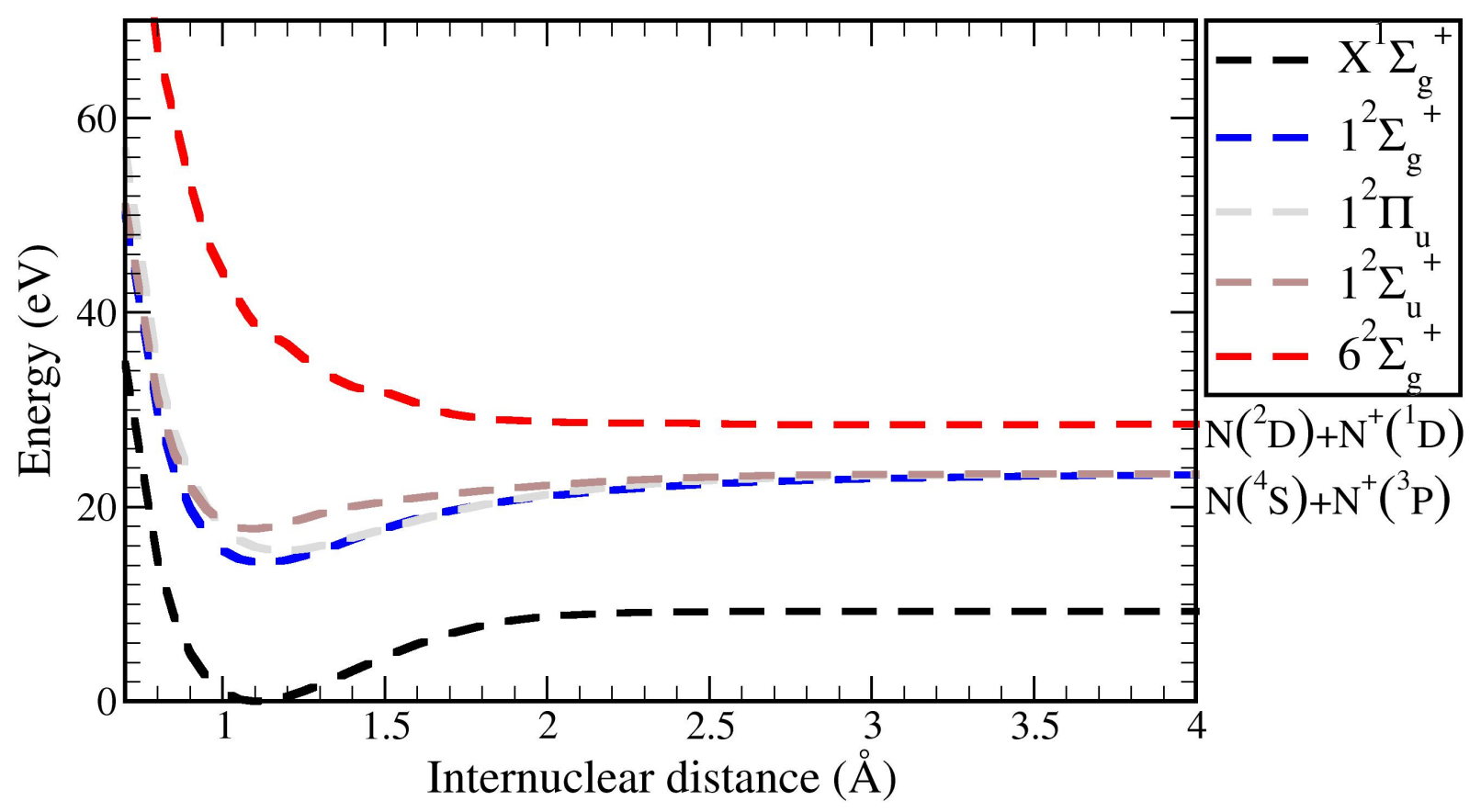

Figure 2: PECs of the $2 \sigma_{g}^{-1}$ state of $\mathrm{N}_{2}$ (red-dashed curve) calculated using CASSCF. For comparison, we also show the ground state (black-dashed curve), $3 \sigma_{g}^{-1}$ (blue-dashed), $1 \pi_{u}^{-1}$ (grey-dashed) and $1^{1} \Sigma_{u}^{+}$(brown-dashed) states as in Figure 1(a). The PECs are plotted with respect to the ground state energy of $\mathrm{N}_{2}$. The dissociation limits are also shown.

The results presented above for the PECs of the ground state and singly ionized states of $\mathrm{N}_{2}$ were obtained using the aug-cc-pVQZ basis set. To test our results for convergence, we also compute the same PECs of these states employing the aug-cc-pVTZ basis set. In Table 1, we show the vertical ionization energy (VIE), adiabatic ionization energy (AIE) and the dissociation energy (DE) of these PECs obtained using the two basis sets. The VIE is the energy difference between the ground state and each singly ionized state of $\mathrm{N}_{2}$ at the 
Table 1: Basis set comparison for valence singly ionized states. DE is computed at an internuclear distance of $4 \AA$. All energies are expressed in $\mathrm{eV}$.

\begin{tabular}{|c|c|cc|cc|c|c|}
\hline \multirow{5}{*}{ Electronic } & \multicolumn{2}{|c|}{ aug-cc-pVTZ } & \multicolumn{2}{c|}{ aug-cc-pVQZ } & Other & Exp. \\
& configuration & CAS & CAS+MRCI & CAS & CAS+MRCI & calculations & \\
\hline \hline \multirow{3}{*}{ VIE } & $3 \sigma_{g}^{-1}$ & 14.31 & 15.35 & 14.30 & 15.39 & - & $15.58^{a}$ \\
& $1 \pi_{u}^{-1}$ & 15.87 & 15.52 & 15.86 & 16.84 & - & $16.926^{a}$ \\
& $2 \sigma_{u}^{-1}$ & 17.47 & 18.53 & 17.75 & 18.60 & - & $18.751^{a}$ \\
& $2 \sigma_{g}^{-1}$ & 36.64 & - & 38.22 & - & $40.5,^{b} 36.9^{c}$ & - \\
\hline \multirow{3}{*}{ AIE } & $3 \sigma_{g}^{-1}$ & 14.30 & 15.34 & 14.29 & 15.39 & $15.347^{d}$ & $15.58^{a}$ \\
& $1 \pi_{u}^{-1}$ & 15.49 & 15.52 & 15.50 & 16.55 & $16.49^{d}$ & $16.693^{a}$ \\
& $2 \sigma_{u}^{-1}$ & 17.47 & 18.53 & 17.74 & 18.59 & $18.558^{d}$ & $18.751^{a}$ \\
\hline \multirow{3}{*}{$\mathrm{DE}$} & $3 \sigma_{g}^{-1}$ & 23.25 & 24.14 & 23.29 & 24.28 & $24.293^{b}$ & $24.31^{a}$ \\
& $1 \pi_{u}^{-1}$ & 23.32 & 24.18 & 23.37 & 24.32 & $24.293^{b}$ & $24.31^{a}$ \\
& $2 \sigma_{u}^{-1}$ & 23.34 & 24.22 & 23.41 & 24.36 & $24.293^{b}$ & $24.31^{a}$ \\
& $2 \sigma_{g}^{-1}$ & 28.35 & - & 28.51 & - & $28.578,{ }^{b} 31.9^{c}$ & - \\
\hline
\end{tabular}

${ }^{a}$ Ref. $48 ;{ }^{b}$ Ref. $16 ;{ }^{c}$ Ref. $24 ;{ }^{d}$ Ref. 22

equilibrium distance of $\mathrm{N}_{2}$. The AIE is the difference between the energy minima of $\mathrm{N}_{2}$ and each one of the singly ionized states. DE corresponds to the final energy of each singly ionized state at the internuclear distance of $4 \AA$. Table 1 shows that we obtain similar results when employing the two different basis sets both in the CASSCF and the CASSCF+MRCI methods. We find that our results are in excellent agreement with the available experimental results for the VIE, AIE and DE obtained in Ref. 48. Moreover, our results for the AIE agree very well with the theoretical results obtained in Ref. 22 when we employ CASSCF+MRCI as the authors in the latter reference. Finally, the dissociation energies we obtain using both CASSCF and the CASSCF+MRCI are in good agreement with the results obtained in Refs. 24,48 and 16.

\section{Core states}

In this section, we present the PECs of the singly ionized states of $\mathrm{N}_{2}$ with one core hole. As discussed previously, we implement a two-step optimization process with restricted core occupancy to compute these states. It is pertinent to mention here that the main motivation of this work is to obtain the electron spectra generated by an FEL pulse also taking into 
account the explicit nuclear motion. In a previous work, ${ }^{11}$ where we treated the nuclear motion phenomenologically through rate equations, we obtained accurate electron spectra using molecular orbitals of $\mathrm{N}_{2}$, i.e. delocalized orbitals. This is in accord with high resolution electron spectroscopy experiments which measured the energy splitting of the $1 \sigma_{g}$ and $1 \sigma_{u}$ molecular core-hole states of $\mathrm{N}_{2} \cdot{ }^{49-52}$ Given the above, here, we obtain the PECs of delocalized molecular core hole states of $\mathrm{N}_{2}$. In addition, to compare with previous studies, ${ }^{26,27}$ we calculate the PECs for core hole states that are localized on a given atomic site of $\mathrm{N}_{2}$.

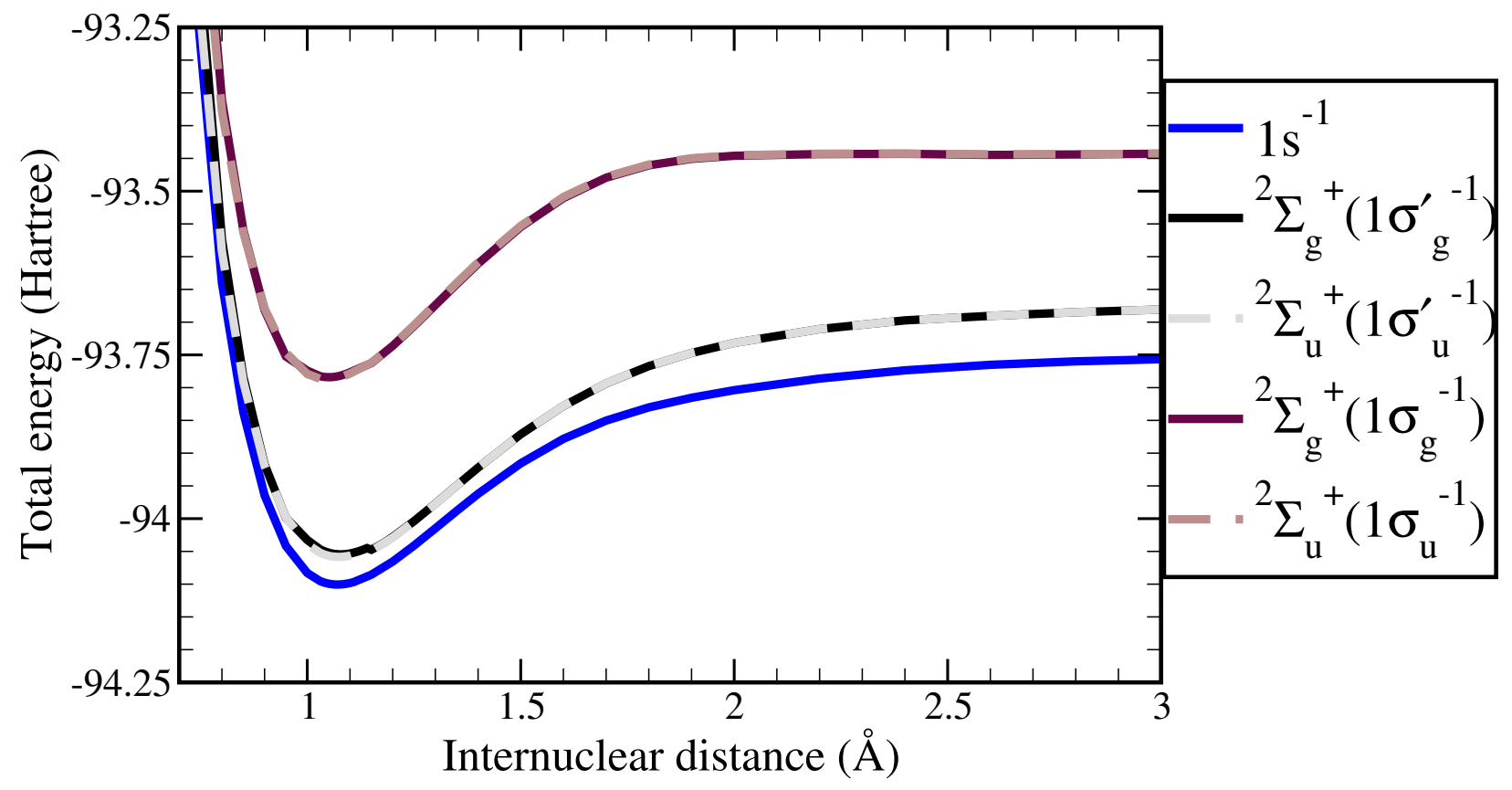

Figure 3: The PECs of singly ionized states of $\mathrm{N}_{2}$ with one core hole.

Following previous work, ${ }^{26,27}$ we calculate the PECs for the singly ionized states of $\mathrm{N}_{2}$ with one core hole that correspond to different types of delocalised orbitals. For one type, we restrict the occupancy of the delocalized molecular orbitals $1 \sigma_{g}$ or $1 \sigma_{u}$ to be equal to 1 , while the occupancy of the other is equal to 2. For the second type, we restrict the occupancy for the pair of $1 \sigma_{g}$ and $1 \sigma_{u}$ orbitals to be equal to 3 ; we denote the resulting delocalized orbitals by $1 \sigma_{g}^{\prime}$ and $1 \sigma_{u}^{\prime}$. Moreover, to compute the PECs using localized orbitals, we proceed as follows. Since our goal is to obtain the lowest energy orbitals localized on one site of $\mathrm{N}_{2}$ we use the $C_{2 v}$ instead of the $D_{2 h}$ symmetry point group in Molpro. The reason is that the 
lowest energy states in $C_{2 v}$, i.e. the $1 \sigma$ and $2 \sigma$ orbitals, do not have the $u$ and $g$ symmetry of the lowest energy $1 \sigma_{g}$ and $1 \sigma_{u}$ orbitals in $D_{2 h}$. As a result, after obtaining the ground state of $\mathrm{N}_{2}$ employing the CASSCF in the $C_{2 v}$ symmetry point group, we localize the two lowest energy orbitals, by applying the Pipek-Mezey localization technique ${ }^{53}$ in Molpro. We label the resulting localized orbitals by 1s. Finally, we restrict the occupancy of either one of the localized core orbitals to 1 , freeze this orbital, and apply CASSCF to optimize the remaining active orbitals.

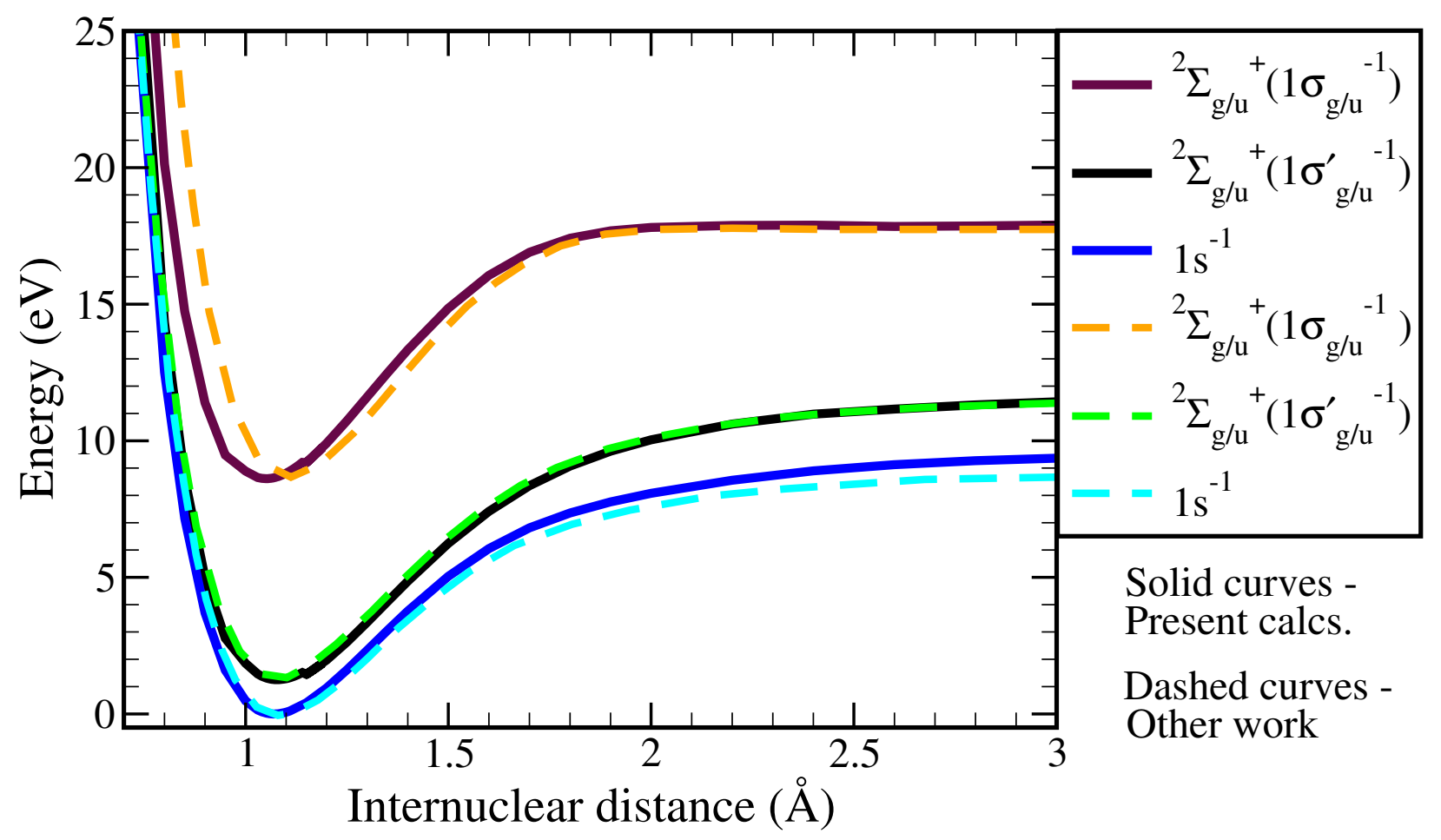

Figure 4: The PECs for the singly ionized states of $\mathrm{N}_{2}$ in comparison with previous work. The PECs depicted by dashed curves are taken from Ref. 27.

Figure 3 shows the PECs of the singly ionized states of $\mathrm{N}_{2}$ with one core hole. For better accuracy ${ }^{55}$ we use the aug-cc-pCVQZ ${ }^{56}$ basis set which is a larger core-augmented as compared to the aug-cc-pVQZ basis set we employed for valence hole states. We note that the particular bases sets in Molpro ${ }^{41,42}$ that we employ in the current work are expressed in terms of contracted gaussians. However, it has been shown that the use of de-contracted basis functions can improve convergence of core orbitals, see Ref. 57. We find that the PECs 
Table 2: Basis set comparison for singly ionized states with core hole. All energies are expressed in $\mathrm{eV}$.

\begin{tabular}{|c|c|c|c|c|c|c|}
\hline & $\begin{array}{c}\text { Electronic } \\
\text { configuration }\end{array}$ & aug-cc-pVTZ & aug-cc-pCVTZ & aug-cc-pCVQZ & $\begin{array}{c}\text { Previous }^{\text {work }^{a}} \\
\text { Exp. }^{b}\end{array}$ \\
\hline \hline \multirow{3}{*}{ AIE } & $\sigma_{g}^{\prime-1}, \sigma_{u}^{\prime-1}$ & $410.28,410.16$ & $410.13,410.02$ & $410.06,409.95$ & $410.8,410.9$ & $409.93,409.82$ \\
& $\sigma_{g}^{-1}, \sigma_{u}^{-1}$ & $417.59,417.49$ & $417.47,417.39$ & $417.42,417.33$ & $418.2,418.3$ & - \\
& $1 \mathrm{~s}^{-1}$ & 409.50 & 409.34 & 409.26 & 409.5 & - \\
\hline \multirow{3}{*}{$D_{e}$} & $\sigma_{g}^{\prime-1}, \sigma_{u}^{\prime-1}$ & $10.43,10.54$ & $10.40,10.51$ & $10.42,10.53$ & 10.31 & - \\
& $\sigma_{g}^{-1}, \sigma_{u}^{-1}$ & $9.35,9.42$ & $9.36,9.41$ & $9.40,9.44$ & 9.00 & - \\
& $1 \mathrm{~s}^{-1}$ & 9.34 & 9.33 & 9.36 & 8.81 & - \\
\hline
\end{tabular}

${ }^{a}$ Ref. $27 ;{ }^{b}$ Ref. 54

of the core localised orbital have lower energy than the PECs for the delocalised orbital sets. Moreover, we find that the PECs of the delocalized orbitals $1 \sigma_{g}^{\prime}, 1 \sigma_{u}^{\prime}$ lie close to each other and the same holds for the PECs for the $1 \sigma_{g}, 1 \sigma_{u}$. However, the PECs of the $1 \sigma_{g}^{\prime}, 1 \sigma_{u}^{\prime}$ differ from the $1 \sigma_{g}, 1 \sigma_{u}$ and have different dissociation limit. In Figure 4 , we compare our results for the PECs of states with one core hole (solid curves) with those obtained in Ref. 27 (dashed curves). As in Ref. 27, we plot the PECs with respect to the minimum energy of the localized core hole state $1 \mathrm{~s}^{-1}$. It is clearly shown that all the PECs quantitatively agree with those reported previously. ${ }^{27}$ The difference in the current work is that the energy minimum of the PECs of $1 \sigma_{g}, 1 \sigma_{u}$ is shifted to shorter bond length.

To test for convergence, we compute the PECs of the singly ionized states of $\mathrm{N}_{2}$ with a core hole employing different basis sets. In Table 2, we show our results for the AIE and the depth of the potential well $\left(\mathrm{D}_{e}\right)$ using the aug-cc-pCVTZ and aug-cc-pCVQZ as well as a smaller valence triple-zeta basis, aug-cc-pVTZ. ${ }^{43}$ Table 2 shows that convergence of our results is already achieved employing the smaller basis set. The depth of the potential well is defined as the energy difference between the dissociation limit and the energy minimum of the PEC. We find that our results are similar for both basis sets. Moreover, we find that our results for $\mathrm{AIE}$ and $\mathrm{D}_{e}$ agree well with the results obtained in the theoretical studies in Ref. 27 where a CASSCF method is employed but with a 6-311G* basis set. Our result for the AIE of the $1 \sigma_{g}^{\prime-1}, 1 \sigma_{u}^{\prime-1}$ states have excellent agreement with experimentally obtained 
results. ${ }^{54}$ For instance, the difference of $110 \mathrm{meV}$ in energy between the $g$ and $u$ symmetry states, observed experimentally (see Table 2) is reproduced very well by our results.

\section{Doubly ionized states}

The ionization of two electrons from any one of the molecular orbitals of $\mathrm{N}_{2}$ results in several ionized states. The final number of doubly ionized states and hence, of PECs increases since we also take the spin into account. If the two electrons are removed from the same molecular orbital the resulting ionized state is a singlet one, while if the two electrons are removed from two different molecular orbitals the resulting states can be either singlet or triplet. Unlike the singly ionized $\mathrm{N}_{2}$ states, we deliver all our calculations for the doubly ionized states using the correlation consistent quintuple basis sets cc-pV5Z and aug-cc-pCV5Z. ${ }^{43}$ These sets were also employed in previous work ${ }^{21,23,58}$ in order to accurately account for correlation effects which are particularly important for doubly ionized states. ${ }^{59}$

\section{Valence states}

To generate doubly ionized states of $\mathrm{N}_{2}$ with two holes in the valence orbitals, we apply the CASSCF method using the cc-pV5Z basis set. For ionized states of $\mathrm{N}_{2}$ with two outer valence holes, we apply the CASSCF method to optimize all ten active orbitals, as we previously did for the ionized states of $\mathrm{N}_{2}$ with one valence hole. To compute the ionized states of $\mathrm{N}_{2}$ with at least one inner valence or core hole, we implement the two-step optimization process that we had previously employed to obtain the ionized states of $\mathrm{N}_{2}$ with a single core hole. We apply the two-step optimization process, within the framework of CASSCF. That is, we first freeze the orbitals where the two electrons are ionizing from and optimize the remaining eight active orbitals. In the second step, we freeze the previously optimized five active occupied orbitals and proceed to optimize the molecular orbitals with missing electrons as well as the three active virtual orbitals. We repeat this process until convergence is achieved.

In Figure 5 we show the PECs of singlet doubly ionized states of $\mathrm{N}_{2}$ with two valence 

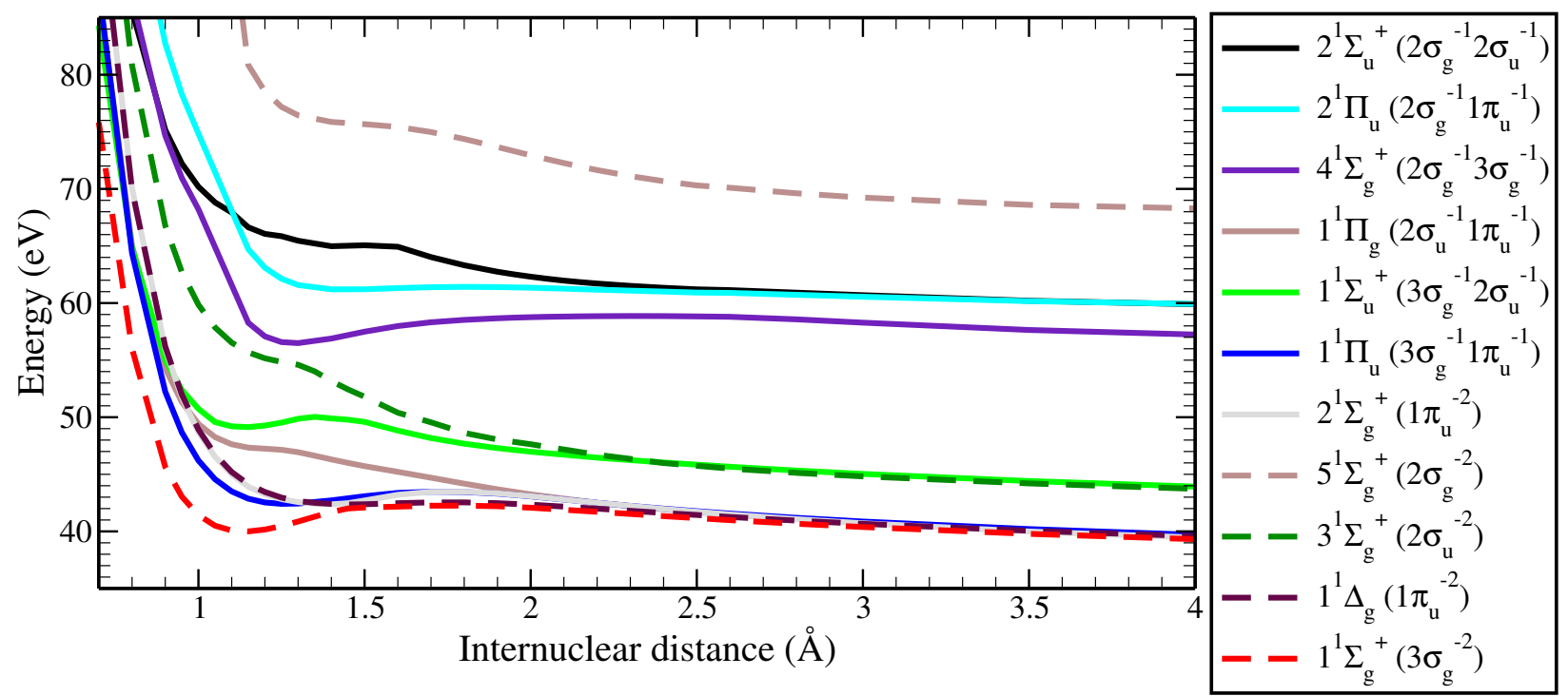

Figure 5: PECs of singlet doubly ionized states of $\mathrm{N}_{2}$ with two valence holes. Dashed (solid) curves represent states where both electrons are removed from the same (different) orbitals.

holes. We find that all PECs converge to five distinct dissociation energies at $4 \AA$. All PECs that have a dissociation energy between 38-44 eV correspond to two electrons missing from outer valence orbitals. Using the energies by Iwayama et al. ${ }^{32}$ of the atomic ion states that corresponds to final dissociation products of $\mathrm{N}_{2}$, we find that doubly ionized states of $\mathrm{N}_{2}$ with two outer valence holes undergo symmetric breaking to $\mathrm{N}^{+}+\mathrm{N}^{+}$. We also find that the states $4^{1} \Sigma_{g}^{+}$and $5^{1} \Sigma_{g}^{+}$undergo an asymmetric dissociation to $\mathrm{N}^{2+}+\mathrm{N}$, while the states $2^{1} \Pi_{u}, 2^{1} \Sigma_{u}^{+}$undergo symmetric break up. In Figure 6, we plot the PECs of triplet doubly ionized states of $\mathrm{N}_{2}$ with two valence holes. The PECs of all triplet $\mathrm{N}_{2}$ states with two outer valence holes have a symmetric bond breaking. The reason is that these triplet states have a DE around $39 \mathrm{eV}$ which can only be accessed by a symmetric bond breaking.

To the best of our knowledge, the PECs of the doubly ionized states of $\mathrm{N}_{2}$ with at least one inner valence hole have not been previously computed. However, there is an experimental prediction by $\mathrm{Wu}$ et $a l .{ }^{21}$ of a $\mathrm{N}_{2}$ state that breaks up asymmetrically with a dissociation energy of $53.9 \mathrm{eV}$ and with a barrier height of $1.3 \mathrm{eV}$. The barrier height is the energy difference between the maximum energy of the barrier of the PEC minus the energy at the dissociation limit. We find that the $2^{3} \Pi_{u}$ state has a dissociation energy of $55.8 \mathrm{eV}$ and a 


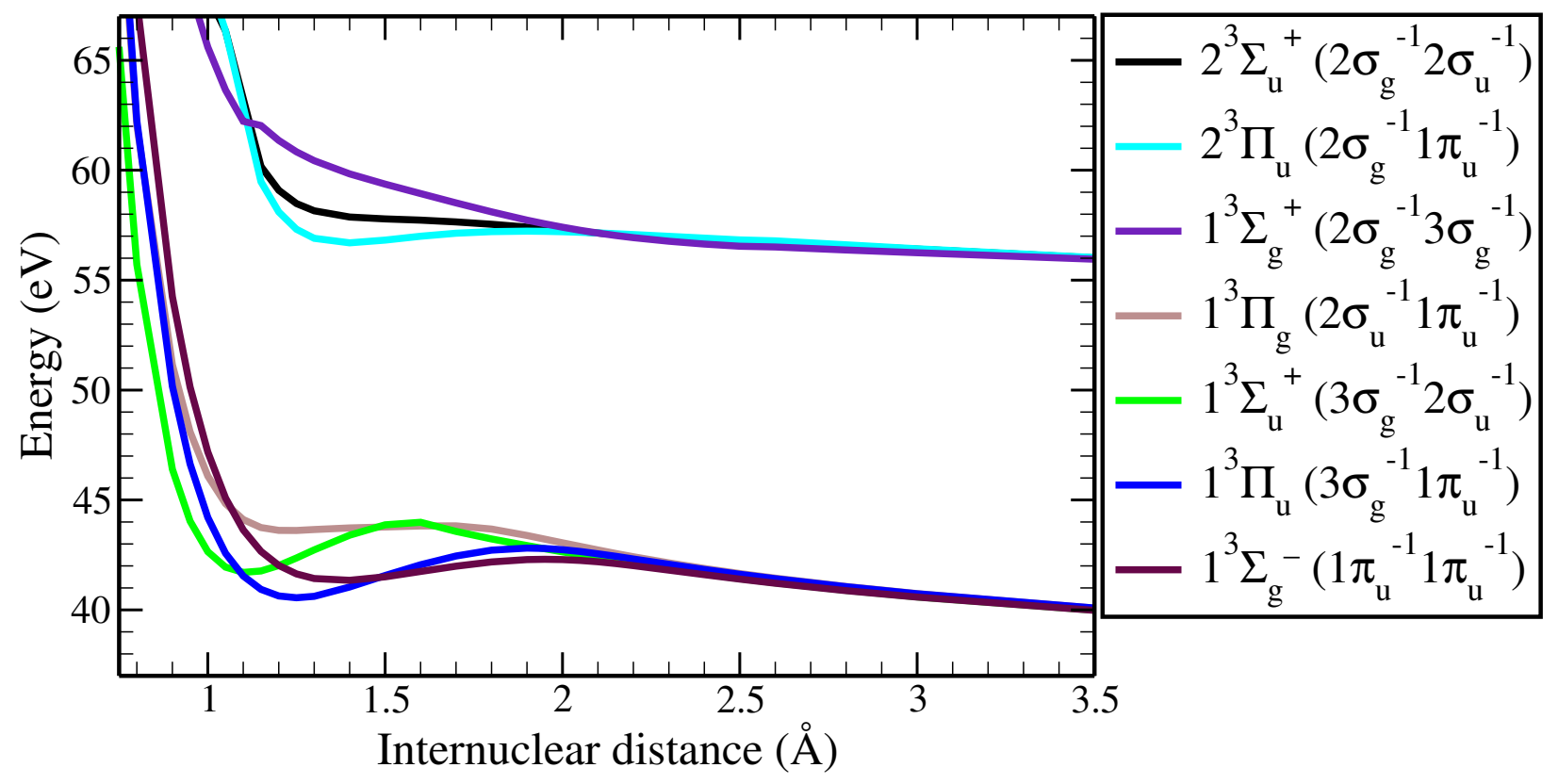

Figure 6: PECs of triplet doubly ionized states of $\mathrm{N}_{2}$ with two valence holes.

barrier height of $1.4 \mathrm{eV}$. Hence, our results suggest that the state observed experimentally is the $2^{3} \Pi_{u}$ state.

Next, to compare our results with previous theoretical work, ${ }^{21,23,58}$ we also employ the CASSCF+MRCI method in order to compute the doubly ionized states of $\mathrm{N}_{2}$ with two outer valence holes. In particular, in Table 3 we show our results and the results of previous work for the vertical, adiabatic and dissociation energies of these states. We find that our results for states $\mathrm{A}$ in Table 3, which correspond to $\mathrm{N}_{2}$ states with two outer valence holes, are in excellent agreement with the values obtained in previous theoretical work. ${ }^{21,58}$ In addition, the calculated VIE is in good agreement with previous experimental findings. ${ }^{32}$ For completeness, we also show in Table 3 our results for $\mathrm{N}_{2}$ states with at least one inner valence hole, denoted by states $\mathrm{B}$. We find that the vertical ionization energies of the states $5^{1} \Sigma_{g}^{+}$and $2^{1} \Sigma_{u}^{+}$are in reasonable agreement with previous experimental results. ${ }^{32}$ We also find that the VIE of the $4^{1} \Sigma_{g}^{+}$state has a difference of $9 \%$ from the experimental result ${ }^{32}$ when we employ CASSCF with 10 active orbitals. However, when we use CASSCF with a larger active space of 12 orbitals $\left(1 \sigma_{g}, 1 \sigma_{u}, 2 \sigma_{g}, 2 \sigma_{u}, 1 \pi_{u x}, 1 \pi_{u y}, 3 \sigma_{g}, 1 \pi_{g x}, 1 \pi_{g y}, 3 \sigma_{u}, 4 \sigma_{g}, 4 \sigma_{u}\right)$, the agreement 
between our result for the VIE and the experimental one is significantly improved, i.e., we find a $3 \%$ difference.

Table 3: Doubly ionized states of $\mathrm{N}_{2}$ with two valence hole. DE is computed at an internuclear distance of $4 \AA$. All energies are expressed in $\mathrm{eV}$. The computations performed with CASSCF involve 10 active orbitals. For the state $4^{1} \Sigma_{g}^{+}$we also perform a CASSCF with 12 active orbitals $(*)$ that yield the values of $65.08 \mathrm{eV}, 58.25 \mathrm{eV}$ and $57.73 \mathrm{eV}$ for VIE, AIE and DE respectively.

\begin{tabular}{|c|c|c|c|c|c|c|c|}
\hline \multirow{2}{*}{$\begin{array}{c}\text { States } \\
\text { A }\end{array}$} & \multirow{2}{*}{$\begin{array}{c}\text { main electronic } \\
\text { configuration }\end{array}$} & \multicolumn{3}{|c|}{ Present calculations (CASSCF+MRCI) } & \multicolumn{2}{|c|}{ Previous work } & \multirow{2}{*}{$\begin{array}{c}\text { Exp. }^{c} \\
\text { VIE }\end{array}$} \\
\hline & & VIE & AIE & $\mathrm{DE}$ & VIE & AIE & \\
\hline 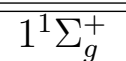 & $\overline{3 \sigma_{g}^{-2}}$ & 42.70 & 42.68 & 42.16 & $\overline{442.5^{a}}$ & $42.6^{b}$ & 43.4 \\
\hline $1^{1} \Delta_{g}^{g}$ & $1 \pi_{u}^{-2}$ & 46.72 & 44.21 & 42.20 & $46.5^{a}$ & $44.09^{b}$ & 46.7 \\
\hline $1^{1} \Pi_{u}$ & $1 \pi_{u}^{-1}, 3 \sigma_{q}^{-1}$ & 45.12 & 44.19 & 42.25 & $44.9^{a}$ & $44.11^{b}$ & 44.7 \\
\hline $1^{1} \Sigma_{u}^{+}$ & $2 \sigma_{u}^{-1}, 3 \sigma_{g}^{-1}$ & 50.57 & 50.53 & 46.06 & $50.4^{a}$ & - & 50.3 \\
\hline $1^{1} \Pi_{g}$ & $1 \pi_{u}^{-1}, 2 \sigma_{u}^{-1}$ & 49.12 & - & 42.26 & $49.0^{a}$ & - & 48.9 \\
\hline $1^{3} \Sigma_{g}^{-}$ & $1 \pi_{u}^{-1}, 1 \pi_{u}^{-1}$ & 45.69 & 43.55 & 42.20 & $45.5^{a}$ & $43.41^{b}$ & - \\
\hline $1^{3} \Pi_{u}^{3}$ & $1 \pi_{u}^{-1}, 3 \sigma_{g}^{-1}$ & 43.59 & 42.76 & 42.25 & $43.4^{a}$ & $42.69^{b}$ & - \\
\hline $1^{3} \Sigma_{u}^{+}$ & $2 \sigma_{u}^{-1}, 3 \sigma_{g}^{-1}$ & 44.11 & 44.11 & 42.18 & $43.9^{a}$ & $44.10^{b}$ & 45.0 \\
\hline $1^{3} \Pi_{g}$ & $1 \pi_{u}^{-1}, 2 \sigma_{u}^{-1}$ & 46.42 & 46.08 & 42.27 & $46.3^{a}$ & $46.06^{c}$ & - \\
\hline & & \multicolumn{3}{|c|}{ Present calculations (CASSCF) } & & & \\
\hline $2^{1} \Sigma_{g}^{+}$ & $1 \pi_{u}^{-2}$ & 44.99 & 42.38 & 39.46 & $46.5^{b}$ & $44.65^{b}$ & 46.8 \\
\hline $3^{1} \Sigma_{g}^{+}$ & $2 \sigma_{u}^{-2}$ & 56.54 & - & 43.73 & - & - & 57.4 \\
\hline States & main electronic & \multicolumn{3}{|c|}{ Present calculations (CASSCF) } & \multicolumn{2}{|c|}{ Previous work } & Exp. ${ }^{c}$ \\
\hline $\mathrm{B}$ & configuration & VIE & AIE & $\mathrm{DE}$ & VIE & AIE & VIE \\
\hline $4^{1} \Sigma_{g}^{+}$ & $2 \sigma_{g}^{-1}, 3 \sigma_{g}^{-1}$ & $61.55 / 65.08^{*}$ & $56.49 / 58.25^{*}$ & $57.24 / 57.73^{*}$ & - & - & 67.4 \\
\hline $5^{1} \Sigma_{g}^{+}$ & $2 \sigma_{g}^{-2}$ & 92.33 & - & 68.28 & - & - & 94.8 \\
\hline $2^{1} \Pi_{u}^{s}$ & $1 \pi_{u}^{-1}, 2 \sigma_{g}^{-1}$ & 68.08 & 61.20 & 59.91 & - & - & - \\
\hline $2^{1} \Sigma_{u}^{+}$ & $2 \sigma_{u}^{-1}, 2 \sigma_{g}^{-1}$ & 67.92 & 64.97 & 59.90 & - & - & 70.8 \\
\hline $1^{3} \Sigma_{g}^{+}$ & $2 \sigma_{g}^{-1}, 3 \sigma_{g}^{-1}$ & 62.22 & - & 55.73 & - & - & - \\
\hline $2^{3} \Pi_{u}$ & $1 \pi_{u}^{-1}, 2 \sigma_{g}^{-1}$ & 62.91 & 56.69 & 55.77 & - & - & - \\
\hline $2^{3} \Sigma_{u}^{+}$ & $2 \sigma_{u}^{-1}, 2 \sigma_{g}^{-1}$ & 63.25 & - & 55.56 & - & - & - \\
\hline
\end{tabular}

${ }^{a}$ Ref. 58; ${ }^{b}$ Ref. $21 ;{ }^{c}$ Ref. 32

\section{Core states}

We now compute the PECs for the doubly ionized states of $\mathrm{N}_{2}$ with one or two core holes in the large core-valence augmented quintuple zeta (aug-cc-pCV5Z) basis set. ${ }^{56}$

Previous theoretical studies ${ }^{60,61}$ have only addressed the energy of $\mathrm{N}_{2}$ states with two core holes at equilibrium distance of the ground state of $\mathrm{N}_{2}$. Hence, to the best of our knowledge, 
the PECs shown in Figure 7 and Figure 8 have not been previously reported. To compute the PECs for $\mathrm{N}_{2}$ states with one or two core holes we use the two-step optimization process within the framework of CASSCF, which we also employed to compute the PECs of doubly ionized $\mathrm{N}_{2}$ states with an inner valence hole. Namely, first, we freeze the orbital(s) with holes while simultaneously restricting its (their) occupancy. Next, we optimize the previously frozen orbitals while now freezing all the remaining active occupied orbitals.

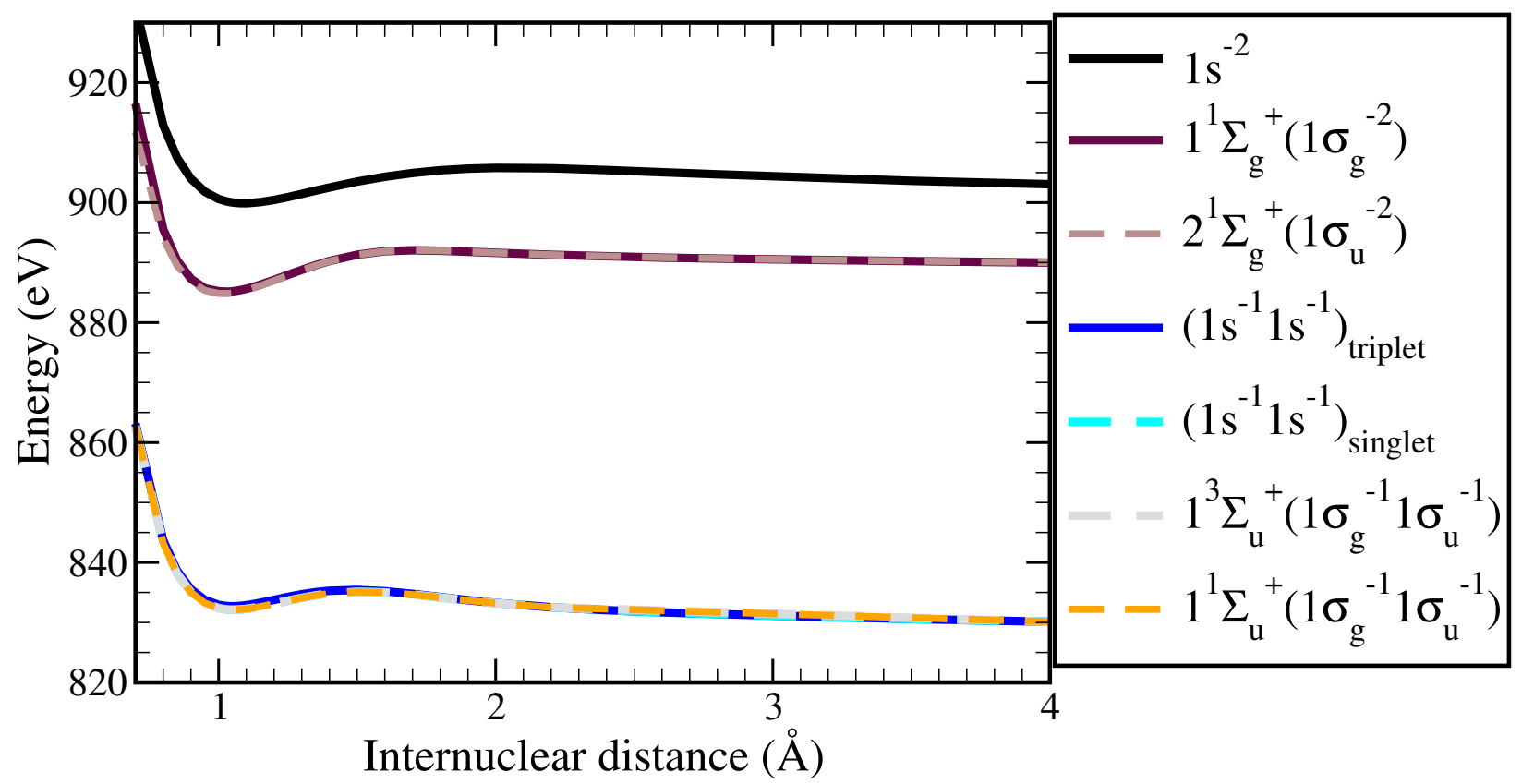

Figure 7: PECs for the doubly ionized states of $\mathrm{N}_{2}$ with two core holes. The state $1 \mathrm{~s}^{-2}$ refers to the removal of two electrons from the same site of localized orbital $1 \mathrm{~s}$. The state $\left(1 \mathrm{~s}^{-1}\right.$ $1 \mathrm{~s}^{-1}$ ) refers to two electrons being removed from localized 1s orbital from different sites.

As with the singly ionized states of $\mathrm{N}_{2}$ with one core hole, we compute the PECs of $\mathrm{N}_{2}$ states with two core holes using both localized and delocalized core orbitals. Figure 7 shows the calculated doubly ionized states of $\mathrm{N}_{2}$ with two core holes with respect to the ground state energy of $\mathrm{N}_{2}$. As expected, we find that the PECs for $1 \mathrm{~s}^{-2}, 1^{1} \Sigma_{g}^{+}$and $2^{1} \Sigma_{g}^{+}$states, which correspond to two electrons missing from the same orbital, are higher in energy than the PECs of all other states where electrons are missing from different orbitals. Figure 8 shows the PECs for the singlet and triplet states of $\mathrm{N}_{2}$ with one core and one valence electron missing. Comparing Figures 8(a) with $8(\mathrm{~b})$ we find that the states with one core and one 
inner valence hole $\left(3 \Sigma_{g / u}^{+}\right)$have the same DE for the singlet and triplet spin symmetries. There is also a marked energy splitting between the $3 \Sigma^{+}$gerade and ungerade states as compared to all other core ionized states presented in Figure. 8. Moreover, we find that a common feature among the PECs of doubly ionized states of $\mathrm{N}_{2}$ with one or two core holes is the existence of an energy minimum and a maximum, i.e. barrier, for increasing internuclear distance.

Previous work ${ }^{60}$ on the double core hole states of $\mathrm{N}_{2}$ reported an ionization energy of 901.1, 835.8 and $836.4 \mathrm{eV}$ for the $1 s^{-2}, 1 s^{-1} 1 s^{-1}$ singlet and triplet states, respectively, using localized orbitals. In the present work, we obtain vertical ionization energies at $1.10 \AA$, of $899.9 \mathrm{eV}, 832.8 \mathrm{eV}$ and $832.3 \mathrm{eV}$ for the corresponding states using localized orbitals. The difference between our results and those reported previously ${ }^{60}$ can be attributed to the different basis sets employed. Specifically, we employ an aug-cc-pCV5Z basis set while a less accurate cc-pVTZ basis set is employed in Ref. 60. For states with two core holes in the $1 \sigma_{g}$ and $1 \sigma_{u}$ orbitals we find the vertical ionization energy to be equal to $885.6 \mathrm{eV}$ and $885.4 \mathrm{eV}$ respectively. The measured ionization energy of $902.55 \mathrm{eV}$ reported in Ref. 61 agrees better with the vertical ionization energy obtained from the localized orbital $(899.9 \mathrm{eV})$ as compared to the delocalized $1 \sigma_{g}(885.6 \mathrm{eV})$ and $1 \sigma_{u}(885.4 \mathrm{eV})$ set of orbitals.

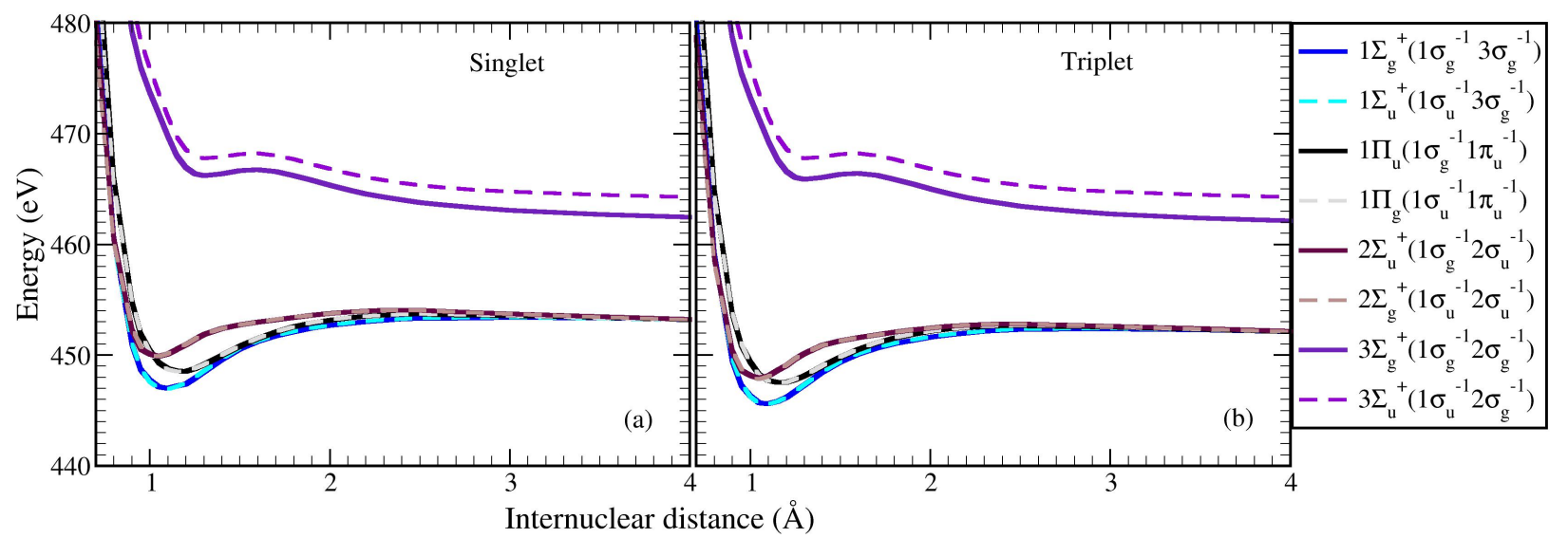

Figure 8: PECs for the doubly ionized states of $\mathrm{N}_{2}$ with a single core hole and a valence hole. 


\section{Summary}

We have presented the PECs for a number of singly and doubly ionized states of $\mathrm{N}_{2}$. To the best of our knowledge, the PECs for doubly ionized states of $\mathrm{N}_{2}$ with one or more inner valence or core holes have not been previously reported. In order to obtain the PECs for singly or doubly ionized states of $\mathrm{N}_{2}$ with outer valence holes, we have employed all-electron CASSCF method with ten active orbitals. This has been followed by MRCI calculations and compared with previous theoretical or experimental results. To obtain the PECs for singly ionized $\mathrm{N}_{2}$ states with a core hole as well as for doubly ionized states with at least one inner valence or core hole, we have employed a two-step optimization process within the framework of CASSCF. In the first step, we freeze the orbitals with holes, restrict their occupancy, and optimize the remaining active orbitals. In the second step, we optimize the orbitals with holes and freeze the rest of the active orbitals that have been optimized in the first step. We repeat this process until convergence is achieved. A similar two-step optimization process has been

previously employed in the context of singly ionized states of $\mathrm{N}_{2}$ with a single core hole. ${ }^{25-27}$ In this work, we have demonstrated the general applicability of this technique to the doubly ionized states of $\mathrm{N}_{2}$. Where ever applicable, our calculations are in good agreement with previously reported calculations and/or experimental results. Specifically, we have computed and confirmed that the doubly ionized state with at least one inner valence hole that has a barrier height of $\sim 1.3 \mathrm{eV}$ and undergo asymmetric charge separation upon dissociation is the $2^{3} \Pi_{u}$ state. The PECs presented in this work are expected to serve as a reference for explicitly accounting for nuclear motion during the interaction of free electron laser pulses interacting with diatomic molecules, such as $\mathrm{N}_{2}$. It is important to note that the two-step optimization technique described in this work can be easily extended to calculate any core or inner valence ionized state, with any number of holes. Although, we have specifically applied this scheme to molecular nitrogen, the optimization technique outlined in the present work can be universally applied to any other single, double or triple bonded polyatomic system. 


\section{Acknowledgement}

D.B and A.E acknowledge the use of the Myriad computational resources at UCL. This work was funded by the Leverhulme Trust Research Project Grant No. 2017-376. The authors thank Prof. Alexandre Rocha for useful discussion concerning the two-step CASSCF method and valuable suggestions.

\section{References}

(1) Geneaux, R.; Marroux, H. J.; Guggenmos, A.; Neumark, D. M.; Leone, S. R. Transient absorption spectroscopy using high harmonic generation: a review of ultrafast X-ray dynamics in molecules and solids. Philos. Trans. R. Soc., A 2019, 377, 20170463.

(2) McNeil, B. W.; Thompson, N. R. X-ray free-electron lasers. Nat. Photonics 2010, 4, $814-821$.

(3) Krausz, F.; Ivanov, M. Attosecond physics. Rev. Mod. Phys. 2009, 81, 163.

(4) Uiberacker, M. et al. Attosecond real-time observation of electron tunnelling in atoms. Nature 2007, 446, 627-632.

(5) Schultze, M. et al. Delay in photoemission. Science 2010, 328, 1658-1662.

(6) Ranitovic, P.; Hogle, C. W.; Rivière, P.; Palacios, A.; Tong, X.-M.; Toshima, N.; González-Castrillo, A.; Martin, L.; Martín, F.; Murnane, M. M.; Kapteyn, H. Attosecond vacuum UV coherent control of molecular dynamics. Proc. Natl. Acad. Sci. 2014, 111, $912-917$.

(7) Calegari, F.; Ayuso, D.; Trabattoni, A.; Belshaw, L.; De Camillis, S.; Anumula, S.; Frassetto, F.; Poletto, L.; Palacios, A.; Decleva, P.; Greenwood, J. B.; Martin, F.; Nisoli, M. Ultrafast electron dynamics in phenylalanine initiated by attosecond pulses. Science 2014, 346, 336-339. 
(8) Kraus, P. M.; Mignolet, B.; Baykusheva, D.; Rupenyan, A.; Hornỳ, L.; Penka, E. F.; Grassi, G.; Tolstikhin, O. I.; Schneider, J.; Jensen, F.; Madsen, L. B.; Bandrauk, A. D.; Remacle, F.; Worner, H. J. Measurement and laser control of attosecond charge migration in ionized iodoacetylene. Science 2015, 350, 790-795.

(9) Jahnke, T. et al. Ultrafast energy transfer between water molecules. Nat. Phys. 2010, 6, 139-142.

(10) Banks, H.; Little, D.; Tennyson, J.; Emmanouilidou, A. Interaction of molecular nitrogen with free-electron-laser radiation. Phys. Chem. Chem. Phys. 2017, 19, 19794-19806.

(11) Banks, H. I.; Little, D. A.; Emmanouilidou, A. Multiple core-hole formation by freeelectron laser radiation in molecular nitrogen. J. Phys. B: At., Mol. Opt. Phys. 2018, $51,095001$.

(12) Banks, H. I.; Hadjipittas, A.; Emmanouilidou, A. Carbon monoxide interacting with free-electron-laser pulses. J. Phys. B: At., Mol. Opt. Phys. 2020, 53, 225602.

(13) Nagy, O.; Ballance, C.; Berrington, K.; Burke, P.; McLaughlin, B. Vibrational excitation of the $\mathrm{N}_{2}^{+}$first negative $(0,0),(1,0)$ and $(2,0)$ bands by electron impact: a theoretical study using the R-matrix approach. J. Phys. B: At., Mol. Opt. Phys. 1999, 32, L469.

(14) Polák, R.; Fišer, J. A CASSCF/icMRCI study of the electric field gradient in low-lying electronic states of $\mathrm{N}_{2}^{+} / \mathrm{N}_{2}$. Chem. Phys. 2003, 290, 177-188.

(15) Gagnon, E.; Ranitovic, P.; Tong, X.-M.; Cocke, C. L.; Murnane, M. M.; Kapteyn, H. C.; Sandhu, A. S. Soft X-ray-driven femtosecond molecular dynamics. Science 2007, 317, $1374-1378$.

(16) Aoto, T.; Ito, K.; Hikosaka, Y.; Shibasaki, A.; Hirayama, R.; Yamamono, N.; Miyoshi, E. Inner-valence states of $\mathrm{N}_{2}^{+}$and the dissociation dynamics studied by threshold photo- 
electron spectroscopy and configuration interaction calculation. J. Chem. Phys. 2006, 124, 234306.

(17) Trabattoni, A.; Klinker, M.; González-Vázquez, J.; Liu, C.; Sansone, G.; Linguerri, R.; Hochlaf, M.; Klei, J.; Vrakking, M.; Martín, F.; Nisoli, M.; Calegari, F. Mapping the dissociative ionization dynamics of molecular nitrogen with attosecond time resolution. Phys. Rev. X 2015, 5, 041053.

(18) Roothaan, C. C.; Detrich, J.; Hopper, D. G. An improved MCSCF method. Int. J. Quantum Chem. 1979, 16, 93-101.

(19) Werner, H. J.; Meyer, W. A quadratically convergent MCSCF method for the simultaneous optimization of several states. J. Chem. Phys. 1981, 74, 5794-5801.

(20) Lengsfield III, B. H.; Liu, B. A second order MCSCF method for large CI expansions. $J$. Chem. Phys. 1981, 75, 478-480.

(21) Wu, Z.; Wu, C.; Liu, X.; Deng, Y.; Gong, Q.; Song, D.; Su, H. Double ionization of nitrogen from multiple orbitals. J. Phys. Chem. A 2010, 114, 6751-6756.

(22) Plésiat, E.; Decleva, P.; Martín, F. Vibrational branching ratios in the photoelectron spectra of $\mathrm{N}_{2}$ and CO: Interference and diffraction effects. Phys. Chem. Chem. Phys. 2012, 14, 10853-10871.

(23) Pandey, A.; Bapat, B.; Shamasundar, K. Charge symmetric dissociation of doubly ionized $\mathrm{N}_{2}$ and CO molecules. J. Chem. Phys. 2014, 140, 034319.

(24) Eckstein, M.; Yang, C.-H.; Kubin, M.; Frassetto, F.; Poletto, L.; Ritze, H.-H.; Vrakking, M. J.; Kornilov, O. Dynamics of $\mathrm{N}_{2}$ dissociation upon inner-valence ionization by wavelength-selected XUV pulses. J. Phys. Chem. Lett. 2015, 6, 419-425.

(25) Rocha, A. B.; de Moura, C. E. The problem of hole localization in inner-shell states 
of $\mathrm{N}_{2}$ and $\mathrm{CO}_{2}$ revisited with complete active space self-consistent field approach. $J$. Chem. Phys. 2011, 135, 224112.

(26) de Moura, C. E.; Oliveira, R. R.; Rocha, A. B. Transition energy and potential energy curves for ionized inner-shell states of $\mathrm{CO}, \mathrm{O}_{2}$ and $\mathrm{N}_{2}$ calculated by several inner-shell multiconfigurational approaches. J. Mol. Model. 2013, 19, 2027-2033.

(27) Corral, I.; Gonzalez-Vazquez, J.; Martín, F. Potential energy surfaces of core-hole and shake-up states for dissociative ionization studies. J. Chem. Theory Comput. 2017, 13, $1723-1736$.

(28) Besley, N. A.; Gilbert, A. T.; Gill, P. M. Self-consistent-field calculations of core excited states. J. Chem. Phys. 2009, 130, 124308.

(29) Loos, P.-F.; Assfeld, X. Core-ionized and core-excited states of macromolecules. Int. J. Quantum Chem. 2007, 10\%, 2243-2252.

(30) Rocha, A. B. Potential curves for inner-shell states of CO calculated at multiconfigurational self-consistent field level. J. Chem. Phys. 2011, 134, 024107.

(31) Carravetta, V.; Ågren, H. Symmetry breaking and hole localization in multiple core electron ionization. J. Phys. Chem. A 2013, 117, 6798-6802.

(32) Iwayama, H.; Kaneyasu, T.; Hikosaka, Y.; Shigemasa, E. Stability and dissociation dynamics of $\mathrm{N}_{2}^{++}$ions following core ionization studied by an Auger-electron-photoion coincidence method. J. Chem. Phys. 2016, 145, 034305.

(33) Roos, B. O. Lecture notes in Quantum Chemistry; Springer, 1992; pp 177-254.

(34) Malmqvist, P.-A.; Roos, B. O. The CASSCF state interaction method. Chem. Phys. Lett. 1989, 155, 189-194.

(35) Schmidt, M. W.; Gordon, M. S. The construction and interpretation of MCSCF wavefunctions. Annu. Rev. Phys. Chem. 1998, 49, 233-266. 
(36) Olsen, J. The CASSCF method: A perspective and commentary. Int. J. Quantum Chem. 2011, 111, 3267-3272.

(37) Werner, H.-J.; Knowles, P. J. A second order multiconfiguration SCF procedure with optimum convergence. J. Chem. Phys. 1985, 82, 5053-5063.

(38) Knowles, P. J.; Werner, H.-J. An efficient second-order MC SCF method for long configuration expansions. Chem. Phys. Lett. 1985, 115, 259-267.

(39) Kreplin, D. A.; Knowles, P. J.; Werner, H.-J. Second-order MCSCF optimization revisited. I. Improved algorithms for fast and robust second-order CASSCF convergence. J. Chem. Phys. 2019, 150, 194106.

(40) Kreplin, D. A.; Knowles, P. J.; Werner, H.-J. MCSCF optimization revisited. II. Combined first-and second-order orbital optimization for large molecules. The Journal of chemical physics 2020, 152, 074102.

(41) Werner, H.-J.; Knowles, P. J.; Knizia, G.; Manby, F. R.; Schütz, M. Molpro: a generalpurpose quantum chemistry program package. Wiley Interdiscip. Rev.: Comput. Mol. Sci. 2012, 2, 242-253.

(42) Werner, H.-J.; Knowles, P. J.; Manby, F. R.; Black, J. A.; Doll, K.; Heßelmann, A.; Kats, D.; Köhn, A.; Korona, T.; Kreplin, D. A., et al. The Molpro quantum chemistry package. J. Chem. Phys. 2020, 152, 144107.

(43) Dunning Jr, T. H. Gaussian basis sets for use in correlated molecular calculations. I. The atoms boron through neon and hydrogen. J. Chem. Phys. 1989, 90, 1007-1023.

(44) Werner, H.-J.; Knowles, P. J. An efficient internally contracted multiconfigurationreference configuration interaction method. J. Chem. Phys. 1988, 89, 5803-5814.

(45) Knowles, P. J.; Werner, H.-J. An efficient method for the evaluation of coupling coefficients in configuration interaction calculations. Chem. Phys. Lett. 1988, 145, 514-522. 
(46) Knowles, P. J.; Werner, H.-J. Internally contracted multiconfiguration-reference configuration interaction calculations for excited states. Theor. Chim. Acta 1992, 84, 95-103.

(47) Frost, D.; McDowell, C. The dissociation energy of the nitrogen molecule. Proc. R. Soc. London, Ser. A 1956, 236, 278-284.

(48) Baltzer, P.; Larsson, M.; Karlsson, L.; Wannberg, B.; Göthe, M. C. Inner-valence states of $\mathrm{N}_{2}^{+}$studied by UV photoelectron spectroscopy and configuration-interaction calculations. Phys. Rev. A 1992, 46, 5545.

(49) Hergenhahn, U.; Kugeler, O.; Rüdel, A.; Rennie, E. E.; Bradshaw, A. M. Symmetryselective observation of the $\mathrm{N} 1 \mathrm{~s}$ shape resonance in $\mathrm{N}_{2}$. J. Phys. Chem. A 2001, 105, $5704-5708$.

(50) Ueda, K.; Püttner, R.; Cherepkov, N.; Gel'mukhanov, F.; Ehara, M. High resolution X-ray photoelectron spectroscopy on nitrogen molecules. Eur. Phys. J.: Spec. Top. 2009, 169, 95-107.

(51) Ueda, K. To be or not to be localized. Science 2008, 320, 884-885.

(52) Schöffler, M.; Titze, J.; Petridis, N.; Jahnke, T.; Cole, K.; Schmidt, L. P. H.; Czasch, A.; Akoury, D.; Jagutzki, O.; Williams, J., et al. Ultrafast probing of core hole localization in $\mathrm{N}_{2}$. Science 2008, 320, 920-923.

(53) Pipek, J.; Mezey, P. G. A fast intrinsic localization procedure applicable for abinitio and semiempirical linear combination of atomic orbital wave functions. J. Chem. Phys. 1989, 90, 4916-4926.

(54) Alagia, M.; Richter, R.; Stranges, S.; Agåker, M.; Ström, M.; Söderström, J.; Såthe, C.; Feifel, R.; Sorensen, S.; De Fanis, A., et al. Core level ionization dynamics in small 
molecules studied by x-ray-emission threshold-electron coincidence spectroscopy. Phys. Rev. A 2005, 71, 012506.

(55) Ambroise, M. A.; Jensen, F. Probing basis set requirements for calculating core ionization and core excitation spectroscopy by the $\Delta$ self-consistent-field approach. J. Chem. Theory Comput. 2018, 15, 325-337.

(56) Kendall, R. A.; Dunning Jr, T. H.; Harrison, R. J. Electron affinities of the first-row atoms revisited. Systematic basis sets and wave functions. J. Chem. Phys. 1992, 96, 6796-6806.

(57) Sarangi, R.; Vidal, M. L.; Coriani, S.; Krylov, A. I. On the basis set selection for calculations of core-level states: Different strategies to balance cost and accuracy. Mol. Phys. 2020, 118, e1769872.

(58) Pandey, A.; Saha, K.; Bapat, B.; Kumar, P.; Banerjee, S.; Subramanian, K. Probing high-lying $\mathrm{N}_{2}^{++}$and $\mathrm{C}_{O}^{++}$states via energy-selective fragment spectra. J. Phys. B: At., Mol. Opt. Phys. 2016, 49, 135102.

(59) Bennett, F. R. Accurate ab initio potential energy functions for doubly charged diatomics. J. Chem. Phys. 1995, 103, 3613-3620.

(60) Tashiro, M.; Ehara, M.; Fukuzawa, H.; Ueda, K.; Buth, C.; Kryzhevoi, N. V.; Cederbaum, L. S. Molecular double core hole electron spectroscopy for chemical analysis. J. Chem. Phys. 2010, 132, 184302.

(61) Lablanquie, P. et al. Properties of hollow molecules probed by single-photon double ionization. Phys. Rev. Letters 2011, 106, 063003. 


\section{Graphical TOC Entry}

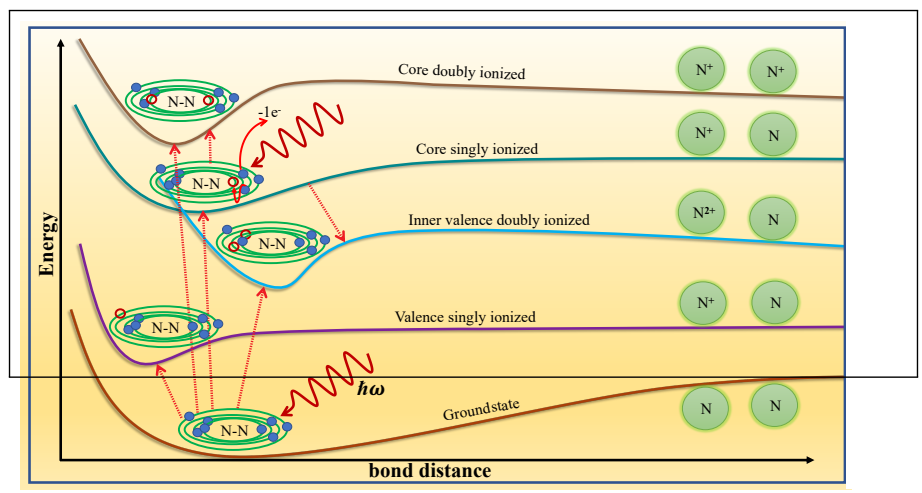

